\title{
Clifford structures in noncommutative geometry and the extended scalar sector
}

\author{
Maxim A. Kurkov ${ }^{1,2,3, *}$ and Fedele Lizzi ${ }^{2,3,4, \dagger}$ \\ ${ }^{1}$ Centro de Matemática, Computação e Cognição, Universidade Federal do ABC, \\ 09210-580 Santo André, São Paulo, Brazil \\ ${ }^{2}$ Dipartimento di Fisica “Ettore Pancini," Università di Napoli Federico II, 80125 Napoli, Italy \\ ${ }^{3}$ INFN, Sezione di Napoli, 80125 Napoli, Italy \\ ${ }^{4}$ Departament de Física Quàntica i Astrofísica and Institut de Cíencies del Cosmos (ICCUB), \\ Universitat de Barcelona, 08028 Barcelona, Spain
}

(Received 30 January 2018; published 30 April 2018)

\begin{abstract}
We consider aspects of the noncommutative approach to the standard model based on the spectral action principle. We show that as a consequence of the incorporation of the Clifford structures in the formalism, the spectral action contains an extended scalar sector, with respect to the minimal standard model. This may have interesting phenomenological consequences. Some of these new scalar fields carry both weak isospin and color indices. We calculate the new terms in spectral action due to the presence of these fields. Our analysis demonstrates that the fermionic doubling in the noncommutative geometry is not just a presence of spurious degrees of freedom, but it is an interesting and peculiar property of the formalism, which leads to physically valuable conclusions. Some of the new fields do not contribute to the physical fermionic action, but they appear in the bosonic spectral action. Their contributions to the Dirac operator correspond to couplings with the spurious fermions, which are projected out.
\end{abstract}

DOI: 10.1103/PhysRevD.97.085024

\section{INTRODUCTION}

The standard model of particle interactions can be efficiently described by a particular noncommutative geometry: an "almost commutative geometry." Over the years the model has been developing both in its mathematical and physical aspects. Its mathematical framework has its roots in a global view [1-4] of geometry based on the spectral properties of operators. The applications of this point of view to geometry are quite startling; the standard reference of the model in its modern version is [5]; for a recent review see [6]. The model has predictive power, although it is premature to consider it a fully fledged theory to confront with experiment, with prediction with a significative number of digits. Its main success is in the description of the symmetries of the model; very few Yang-Mills models can be described by a noncommutative geometry (NCG), but the standard model and a few more can. The Higgs field emerges naturally as an intermediate boson corresponding to the noncommutative part of the model, of

\footnotetext{
*max.kurkov@gmail.com

†fedele.lizzi@na.infn.it
}

Published by the American Physical Society under the terms of the Creative Commons Attribution 4.0 International license. Further distribution of this work must maintain attribution to the author(s) and the published article's title, journal citation, and DOI. Funded by SCOAP ${ }^{3}$. a par with photons, $W, Z$, and gluons. The actions for fermions and bosons are firmly based on the spectral properties of a generalized Dirac operator [7], ${ }^{1}$ and the procedure is capable of obtaining numbers such as the mass of the Higgs boson. The numbers produced in [5], although encouraging, are not in agreement with present data; in particular, the model requires the unification of all couplings at a single energy, and one calculates the Higgs boson mass around $170 \mathrm{GeV}$. Both these aspects are experimentally excluded, and the model can be fixed to allow the physical mass of the Higgs boson [12-21]. Efforts are also undertaken to use the model for other predictions, for example, in [22].

The fact that the calculations made in the present model are encouraging, but not yet comparable with experiment, suggests that some improvement may happen also from the mathematical side. In [23] a noncommutative version of the Clifford symmetry was discussed. One of the remarkable effects of the Clifford requirements is the appearance of scalar fields that are not present in the usual description.

The aim of this paper is to discuss in detail these new fields and their couplings. In particular, we calculate their contribution to the spectral action. The noncommutative

\footnotetext{
${ }^{1}$ It is remarkable that the spectral action is intimately connected to anomalies [8-10], and further development of this observation leads to interesting results beyond the noncommutative geometry [11].
} 
model is by nature Euclidean and exhibits spurious degrees of freedom, known as "fermion doubling" [24]; therefore, for physical applications a Wick (anti)rotation accompanied by an elimination of these spurious degrees of freedom is necessary. We have described this procedure in detail in [25]. Here we find that not all of these extra bosons behave upon this procedure in a standard way: some of the new scalar fields present in the Euclidean Dirac operator are absent in the corresponding (Lorentzian) physical action for fermions.

The paper is organized as follows: in Sec. II we review the noncommutative geometric approach to the standard model, focusing on the modification of the formalism due to an introduction of the Clifford structures proposed in [23]. In Sec. III we introduce the new scalar fields, which come out from the fluctuations of the Dirac operator in the "Clifford-based" approach [23], and discuss their transformation properties upon the action of the gauge group. Section IV is devoted to the bosonic spectral action: we compute the new terms with respect to the "standard" spectral approach [5]. In Sec. V we discuss the physical action derived from this model: we carry out the Wick rotation to the Lorentzian signature and get rid of the spurious degrees of freedom in the fermionic action. The last section contains our conclusions.

\section{THE STANDARD MODEL AS A NONCOMMUTATIVE GEOMETRY}

In this section we sketch the main aspects of the model. We are very brief; the reader familiar with this approach will need this section just to set the notations. First we outline the basic concepts of the spectral triples, which are common for both the standard approach [5] and the Clifford-based [23] approaches; afterwards we discuss the peculiar features of the latter, which differentiate it from the former: the finite dimensional grading $\gamma_{F}$ and the finite dimensional Dirac operator $D_{F}$.

\section{A. The standard spectral triple}

In the spectral approach a geometry is described by a spectral triple [1-3], i.e., a $*$-algebra (possibly noncommutative) realized as bounded operators on a Hilbert space, and a self-adjoint operator that generalizes the Dirac operator. The algebra describes the topology of the space; for the case at hand the Hilbert space describes the matter content and the Dirac operator gives a metric structure and enables the writing of action. Being based on operators all quantities are based on spectra, and, in particular, the actions for bosons and fermions can be written in purely spectral form. Also of fundamental importance are two more operators: the grading and the real structure, which generalize chirality (for the even dimensional case) and charge conjugation. The standard model emerges from this scheme. We briefly describe this approach mainly to set notations, referring to details in the original literature $[5,7]$ or the recent book [6]. We start choosing an algebra that is the product commutative infinite dimensional algebra of continuous functions on the manifold $\mathcal{M}$, which represents the space-time times a noncommutative but finite dimensional matrix algebra,

$$
\mathcal{A}=C(\mathcal{M}) \otimes \mathcal{A}_{F} .
$$

For the standard model the finite algebra is

$$
\mathcal{A}_{F}=\mathbb{C} \oplus \mathbb{H} \oplus \operatorname{Mat}_{3}(\mathbb{C}),
$$

where by $\mathbb{W}$ we indicate quaternions, and by $\mathrm{Mat}_{3}(\mathbb{C})$ three by three complex matrices. Likewise the Hilbert space is the product of usual spinors times a finite dimensional Hilbert space, which contains all physical degrees of freedom,

$$
\mathcal{H}=\operatorname{sp}(\mathcal{M}) \otimes \mathcal{H}_{F}
$$

the generalized Dirac operator (which in the following we simply call Dirac operator) is

$$
\mathcal{D}_{0}=\mathrm{i} \gamma^{\mu} \nabla_{\mu}^{\mathrm{LC}} \otimes 1_{F}+\gamma^{5} \otimes D_{F},
$$

where $\nabla_{\mu}^{\mathrm{LC}}$ is the covariant derivative on the spinor bundle of $\mathcal{M}$, which contains the Levi-Civita spin connection. Gravity in the action is considered background, and is not quantized. A curved background does not however play a major role in this paper, but is useful to retain it, as it enables some simplification in the calculations, as we see in Sec. V.

As we mentioned, there are two more operators that play an important role. They are the grading operator $\Gamma$ and the antiunitary real structure $\mathcal{J}$. The grading operator $\Gamma$ is present in the even dimensional case; it satisfies $\Gamma^{2}=\mathbb{1}$ and is taken to be

$$
\Gamma=\gamma^{5} \otimes \gamma_{F},
$$

where $\gamma^{5}$ is the chirality matrix; i.e., the usual product of all four Dirac's $\gamma^{\mu}$ and $\gamma_{F}$ is an operator acting on $\mathcal{H}_{F}$. It is usually taken to have eigenvalue +1 on left-handed states, and -1 on right-handed one, but other choices are possible and we discuss them later in the paper.

The real structure operator $\mathcal{J}=J \otimes J_{F}$, which is antiunitary in $\mathcal{H}$, enables the definition of the opposite algebra,

$$
\mathcal{A}^{o}=\mathcal{J} \mathcal{A J}^{-1} .
$$

The elements of the triple must satisfy several conditions, which render the space the noncommutative equivalent of a manifold [26]. There are conditions of compatibility between $\Gamma, \mathcal{J}$, and $\mathcal{D}_{0}$ with signs that depend on the dimensions, 


$$
\mathcal{J}^{2}= \pm \mathbb{1}, \quad \mathcal{J} \Gamma= \pm \Gamma \mathcal{J}, \quad \mathcal{J D}_{0}= \pm \mathcal{D}_{0} \mathcal{J} .
$$

The opposite algebra must commute with the algebra (order zero condition),

$$
\left[a, \mathcal{J} b \mathcal{J}^{-1}\right]=0, \quad \forall a, b \in \mathcal{A}
$$

and with one forms (the order one condition),

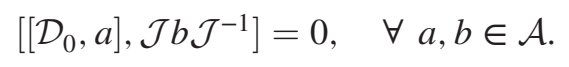

The dimension of $\mathcal{H}_{F}$ in (2.3) is 96. This number is obtained taking into account that there is a lepton left doublet plus two right-handed singlets, and a doublet and two singlets for quarks times three colors. This makes 16 degrees of freedom, times three generations, and times two for particle/antiparticle, sums to 96 . Since the spinor has 4 components the element of the full Hilbert space $\mathcal{H}$ is described by 384 independent complex valued functions. Clearly there is some overcounting, called for historical reasons fermion doubling [24]. We come back to this issue, as well as the fact that the model is at this stage Euclidean, in Sec. V.

We label the elements of $\mathcal{H}_{F}$ according to the basis given by the elementary particles of the standard model (including right-handed neutrinos),

$$
\left(\boldsymbol{\nu}_{R}, \boldsymbol{e}_{R}, \boldsymbol{L}_{L}, \boldsymbol{u}_{R}, \boldsymbol{d}_{\boldsymbol{R}}, \boldsymbol{Q}_{L}, \boldsymbol{\nu}_{R}^{c}, \boldsymbol{e}_{R}^{c}, \boldsymbol{L}_{L}^{c}, \boldsymbol{u}_{R}^{c}, \boldsymbol{d}_{\boldsymbol{R}}^{c}, \boldsymbol{Q}_{L}^{c}\right)
$$

where $Q_{L}$ corresponds to ${ }^{2}$ the quark doublet $\left(\boldsymbol{u}_{L}, \boldsymbol{d}_{L}\right)$ while $L_{L}$ corresponds to the lepton doublet $\left(\boldsymbol{\nu}_{L}, \boldsymbol{e}_{L}\right)$; with the supercript $c$ we indicate the elements of $H_{F}$ that correspond to the antiparticles and by boldface characters we indicate that the elements have to replicated by three generations, for example, $\boldsymbol{e}=(\boldsymbol{e}, \mu, \tau)$ and so on. Quarks have an extra color index, which we omit. Below we use the following notation for matrices action on $\mathcal{H}_{F}$. We define the matrix unity $E_{u_{R} u_{R}}$ to be a matrix whose only nonzero element is an identity matrix in the $\boldsymbol{u}_{R}$ location; likewise for $E_{\boldsymbol{u}_{R} \boldsymbol{d}_{R}}$ it is an off diagonal matrix with nonvanishing entry in the $\boldsymbol{u}_{R} \boldsymbol{d}_{R}$, and so on. In the cases for which a singlet crosses a doublet we assume that, for example, $E_{u_{R}, L_{L}}$ is two identity matrices side by side, or vertically superimposed.

The representation of the algebra is diagonal and with our notation, an element $a=(\lambda, h, m)$ with $\lambda \in \mathbb{C}, h \in \mathbb{U}$ and $m \in \operatorname{Mat}_{3}(\mathbb{C})$ is represented by the matrix, ${ }^{3}$

\footnotetext{
${ }^{2}$ The construction of the product space clarifies in which sense the word "corresponds" is used: see, in particular, the discussion around (5.15).

${ }^{3}$ Here and in the following we omit terms like $\otimes \mathbb{1}_{3}$ when, for example, a complex number acts on a quark, and likewise for doublets etc.
}

$$
\begin{aligned}
a= & \lambda E_{\boldsymbol{u}_{R}, \boldsymbol{u}_{R}}+\lambda^{*} E_{\boldsymbol{d}_{R}, \boldsymbol{d}_{R}}+h E_{\boldsymbol{Q}_{L}, \boldsymbol{Q}_{L}}+\lambda E_{\boldsymbol{\nu}_{R}, \nu_{R}}+\lambda^{*} E_{\boldsymbol{e}_{R}, \boldsymbol{e}_{R}} \\
& +h E_{\boldsymbol{L}_{L}, \boldsymbol{L}_{L}}+m E_{\boldsymbol{u}_{R}^{c}, \boldsymbol{u}_{R}^{c}}+m E_{\boldsymbol{d}_{R}^{c}, \boldsymbol{d}_{R}^{c}}+m E_{\boldsymbol{Q}_{L}^{c}, \boldsymbol{Q}_{L}^{c}}+\lambda E_{\boldsymbol{\nu}_{R}^{c}, \nu_{R}^{c}} \\
& +\lambda E_{\boldsymbol{e}_{R}^{c}, \boldsymbol{e}_{R}^{c}}+\lambda E_{\boldsymbol{L}_{L}^{c}, \boldsymbol{L}_{L}^{c}} .
\end{aligned}
$$

In our notations the real structure $J_{F}$ of the finite spectral triple reads

$$
J_{F}=\left(E_{\boldsymbol{u}_{R}, u_{R}^{c}}+E_{\boldsymbol{d}_{R}, d_{R}^{c}}+E_{\boldsymbol{Q}_{L}, \boldsymbol{Q}_{L}^{c}}+E_{\boldsymbol{\nu}_{R}, \nu_{R}^{c}}+E_{\boldsymbol{e}_{R}, e_{R}^{c}}+E_{L_{L}, \boldsymbol{L}_{L}}\right) c c
$$

where $c c$ is complex conjugation.

So far we have been in the framework of [5]. From now on we focus on the peculiar properties of the construction of [23], which enables us to incorporate the Clifford structures in the finite spectral triple. We refer to the original paper for all the details, and present here just the results.

\section{B. Alternative grading}

The first novelty of the Clifford-based construction is the grading $\gamma_{F}$ of the finite spectral triple, which has the following form:

$$
\begin{aligned}
\gamma_{F}= & -E_{\boldsymbol{u}_{R}, \boldsymbol{u}_{R}}-E_{\boldsymbol{d}_{R}, \boldsymbol{d}_{R}}+E_{\boldsymbol{Q}_{L}, \boldsymbol{Q}_{L}}+E_{\boldsymbol{\nu}_{R}, \boldsymbol{\nu}_{R}}+E_{\boldsymbol{e}_{R}, \boldsymbol{e}_{R}}-E_{\boldsymbol{L}_{L}, \boldsymbol{L}_{L}} \\
& -\left(-E_{\boldsymbol{u}_{R}^{c}, \boldsymbol{u}_{R}^{c}}-E_{\boldsymbol{d}_{R}^{c}, \boldsymbol{d}_{R}^{c}}+E_{\boldsymbol{Q}_{L}^{c}, \boldsymbol{Q}_{L}^{c}}+E_{\boldsymbol{\nu}_{R}^{c}, \nu_{R}^{c}}+E_{\boldsymbol{e}_{R}^{c}, \boldsymbol{e}_{R}^{c}}-E_{\boldsymbol{L}_{L}^{c}, \boldsymbol{L}_{L}^{c}}\right),
\end{aligned}
$$

which differs from the "standard grading" $\gamma_{F}^{\text {st }}$ considered in [5],

$$
\begin{aligned}
\gamma_{F}^{\mathrm{st}}= & -E_{\boldsymbol{u}_{R}, \boldsymbol{u}_{R}}-E_{\boldsymbol{d}_{R}, \boldsymbol{d}_{R}}+E_{\boldsymbol{Q}_{L}, \boldsymbol{Q}_{L}}-E_{\boldsymbol{\nu}_{R}, \nu_{R}}-E_{\boldsymbol{e}_{R}, \boldsymbol{e}_{R}}+E_{\boldsymbol{L}_{L}, \boldsymbol{L}_{L}} \\
& -\left(-E_{\boldsymbol{u}_{R}^{c}, \boldsymbol{u}_{R}^{c}}-E_{\boldsymbol{d}_{R}^{c}, \boldsymbol{d}_{R}^{c}}+E_{\boldsymbol{Q}_{L}^{c}, \boldsymbol{Q}_{L}^{c}}-E_{\boldsymbol{\nu}_{R}^{c}, \nu_{R}^{c}}-E_{\boldsymbol{e}_{R}^{c}, \boldsymbol{e}_{R}^{c}}+E_{\boldsymbol{L}_{L}^{c}, \boldsymbol{L}_{L}^{c}}\right) .
\end{aligned}
$$

The two are connected by the following formula,

$$
\gamma_{F}=(\mathrm{Q}-\mathrm{L}) \gamma_{F}^{\mathrm{st}}
$$

where Q and L stand for the projectors of the quark and leptonic subspaces of $\mathcal{H}_{F}$, respectively,

$$
\begin{aligned}
& \mathrm{Q}=E_{\boldsymbol{u}_{R}, \boldsymbol{u}_{R}}+E_{\boldsymbol{d}_{R}, \boldsymbol{d}_{R}}+E_{\boldsymbol{Q}_{L}, \boldsymbol{Q}_{L}}+E_{\boldsymbol{u}_{R}^{c}, \boldsymbol{u}_{R}^{c}}+E_{\boldsymbol{d}_{R}^{c}, \boldsymbol{d}_{R}^{c}}+E_{\boldsymbol{Q}_{L}^{c}, \boldsymbol{Q}_{L}^{c}}, \\
& \mathrm{~L}=E_{\boldsymbol{\nu}_{R}, \nu_{R}}+E_{\boldsymbol{e}_{R}, \boldsymbol{e}_{R}}+E_{\boldsymbol{L}_{L}, \boldsymbol{L}_{L}}+E_{\boldsymbol{\nu}_{R}^{c}, \nu_{R}^{c}}+E_{\boldsymbol{e}_{R}^{c}, \boldsymbol{e}_{R}^{c}}+E_{\boldsymbol{L}_{L}^{c}, \boldsymbol{L}_{L}^{c}} .
\end{aligned}
$$

\section{The Dirac operator}

Another novelty of the Clifford-based approach is the Dirac operator $D_{F}$, which has the following form, 


$$
\begin{aligned}
D_{F}= & \boldsymbol{\Upsilon}_{\nu} E_{\boldsymbol{\nu}_{R} \boldsymbol{L}_{L}}+\boldsymbol{\Upsilon}_{\boldsymbol{e}} E_{\boldsymbol{e}_{R} \boldsymbol{L}_{L}}+\mathbf{\Upsilon}_{\boldsymbol{u}} E_{\boldsymbol{u}_{R} \boldsymbol{Q}_{L}}+\mathbf{\Upsilon}_{\boldsymbol{d}} E_{\boldsymbol{d}_{R} \boldsymbol{Q}_{L}} \\
& +\boldsymbol{\Omega}^{*} E_{\boldsymbol{\nu}_{R} \boldsymbol{e}_{R}^{c}}+\boldsymbol{\Delta}_{U} E_{\boldsymbol{\nu}_{R}^{c} u_{R}^{c}}+\boldsymbol{\Delta}_{D} E_{\boldsymbol{e}_{R}^{c} d_{R}^{c}}+\boldsymbol{\Delta}_{L} E_{\boldsymbol{L}_{L}^{c} Q_{L}^{c}}+\boldsymbol{K} E_{\boldsymbol{L}_{L} \boldsymbol{u}_{R}^{c}} \\
& +J_{F}\left(\boldsymbol{\Upsilon}_{\nu} E_{\boldsymbol{\nu}_{R} \boldsymbol{L}_{L}}+\mathbf{\Upsilon}_{\boldsymbol{e}} E_{\boldsymbol{e}_{R} \boldsymbol{L}_{L}}+\mathbf{\Upsilon}_{\boldsymbol{u}} E_{\boldsymbol{u}_{R} \boldsymbol{Q}_{L}}+\mathbf{\Upsilon}_{\boldsymbol{d}} E_{\boldsymbol{d}_{R} \boldsymbol{Q}_{L}}\right. \\
& +\boldsymbol{\Omega}^{*} E_{\boldsymbol{\nu}_{R} \boldsymbol{e}_{R}^{c}}+\boldsymbol{\Delta}_{U} E_{\boldsymbol{\nu}_{R}^{c} u_{R}^{c}}+\boldsymbol{\Delta}_{D} E_{\boldsymbol{e}_{R}^{c} d_{R}^{c}}+\boldsymbol{\Delta}_{L} E_{\boldsymbol{L}_{L}^{c} Q_{L}^{c}} \\
& \left.+\boldsymbol{K} E_{\boldsymbol{L}_{L} \boldsymbol{u}_{R}^{c}}\right) J_{F}+\mathbf{\Upsilon}_{R}^{\dagger} E_{\boldsymbol{\nu}_{R} \nu_{R}^{c}}+\text { H.c. }
\end{aligned}
$$

and which is compatible with the new grading $\gamma_{F}$ and other requirements of the approach of [23]. The terms on the first, third, and last lines involve the usual Yukawa couplings and the Majorana mass terms, which are already present in [5].
The second and the fourth lines instead contain novel terms, which are the object of this paper: $\boldsymbol{\Delta}$ and $\mathbf{K}$ provide novel couplings of leptons and quarks; $\boldsymbol{\Omega}$ couples leptons among themselves in the Euclidean action before the projection on the physical subspace. We see later on that the projection to the physical subspace eliminates some of these couplings. It is important that the self-consistency approach of [23] requires, in particular, that

(i) both entries $\boldsymbol{\Delta}_{D}$ and $\boldsymbol{\Delta}_{L}$ must differ from 0 ,

(ii) and at least two out of the three entries $\boldsymbol{\Delta}_{U}, \mathbf{K}$, and $\boldsymbol{\Omega}$ must be different from 0 .

In conclusion we present the explicit matrix form of the Dirac operator $D_{F}$ defined by (2.16),

$$
D_{F}=\left[\begin{array}{cccccc|cccccc}
\cdot & \cdot & \mathbf{\Upsilon}_{\nu} & \boldsymbol{\Delta}_{u}{ }^{*} & \cdot & \cdot & \mathbf{\Upsilon}_{R}^{\dagger} & \mathbf{\Omega}^{*} & \cdot & \cdot & \cdot & \cdot \\
\cdot & \cdot & \mathbf{\Upsilon}_{e} & \cdot & \boldsymbol{\Delta}_{d}{ }^{*} & \cdot & \mathbf{\Omega}^{\dagger} & \cdot & \cdot & \cdot & \cdot & \cdot \\
\mathbf{\Upsilon}_{\nu}^{\dagger} & \mathbf{\Upsilon}_{e}^{\dagger} & \cdot & \cdot & \cdot & \boldsymbol{\Delta}_{L}^{*} & \cdot & \cdot & \cdot & \boldsymbol{K} & \cdot & \cdot \\
\boldsymbol{\Delta}_{u}^{t} & \cdot & \cdot & \cdot & \cdot & \mathbf{\Upsilon}_{u} & \cdot & \cdot & \boldsymbol{K}^{t} & \cdot & \cdot & \cdot \\
\cdot & \boldsymbol{\Delta}_{d}^{t} & \cdot & \cdot & \cdot & \mathbf{\Upsilon}_{d} & \cdot & \cdot & \cdot & \cdot & \cdot & \cdot \\
\cdot & \cdot & \boldsymbol{\Delta}_{L}^{t} & \mathbf{\Upsilon}_{u}^{\dagger} & \mathbf{\Upsilon}_{d}^{\dagger} & \cdot & \cdot & \cdot & \cdot & \cdot & \cdot & \cdot \\
\hline \mathbf{\Upsilon}_{R} & \boldsymbol{\Omega} & \cdot & \cdot & \cdot & \cdot & \cdot & \cdot & \mathbf{\Upsilon}_{\nu}^{*} & \boldsymbol{\Delta}_{u} & \cdot & \cdot \\
\mathbf{\Omega}^{t} & \cdot & \cdot & \cdot & \cdot & \cdot & \cdot & \cdot & \mathbf{\Upsilon}_{e}^{*} & \cdot & \boldsymbol{\Delta}_{d} & \cdot \\
\cdot & \cdot & \cdot & \boldsymbol{K}^{*} & \cdot & \cdot & \mathbf{\Upsilon}_{\nu}^{t} & \mathbf{\Upsilon}_{e}^{t} & \cdot & \cdot & \cdot & \boldsymbol{\Delta}_{L} \\
\cdot & \cdot & \boldsymbol{K}^{\dagger} & \cdot & \cdot & \cdot & \boldsymbol{\Delta}_{u}^{\dagger} & \cdot & \cdot & \cdot & \cdot & \mathbf{\Upsilon}_{u}^{*} \\
\cdot & \cdot & \cdot & \cdot & \cdot & \cdot & \cdot & \boldsymbol{\Delta}_{d}^{\dagger} & \cdot & \cdot & \cdot & \mathbf{\Upsilon}_{d}^{*} \\
\cdot & \cdot & \cdot & \cdot & \cdot & \cdot & \cdot & \cdot & \boldsymbol{\Delta}_{L}^{\dagger} & \mathbf{\Upsilon}_{u}^{t} & \mathbf{\Upsilon}_{d}^{t} & \cdot
\end{array}\right] .
$$

Setting $\boldsymbol{\Delta}_{U, D, L}=0, \boldsymbol{\Omega}=0$, and $\boldsymbol{K}=0$, one obtains the standard $D_{F}$ of [5].

\section{FLUCTUATIONS OF THE DIRAC OPERATOR: FIELDS}

The fluctuated Dirac operator is constructed in the following way,

$$
\mathcal{D}=\mathcal{D}_{0}+\sum_{i} a_{i}\left[\mathcal{D}_{0}, b_{i}\right]+\sum_{i} \mathcal{J} a_{i}\left[\mathcal{D}_{0}, b_{i}\right] \mathcal{J}^{\dagger},
$$

for generic elements $a_{i}, b_{i} \in \mathcal{A}$. Both gauge and scalar fields in the spectral approach come out from these fluctuations. Presence of the new terms (with respect to [5]) in (2.17) indicates new scalar fields, not present in the standard model.

Below we restrict ourselves to the following structures, where the dependence on the generation indices is factorized:

$$
\begin{array}{rlrl}
\mathbf{\Upsilon}_{\nu} & =\hat{Y}_{u} \otimes \tilde{h}_{\nu}^{\dagger}, & \mathbf{\Upsilon}_{e}=\hat{Y}_{d} \otimes h_{e}^{\dagger}, \\
\mathbf{\Upsilon}_{u}=\hat{y}_{u} \otimes \tilde{h}_{u}^{\dagger}, & \mathbf{\Upsilon}_{d}=\hat{y}_{d} \otimes h_{d}^{\dagger}, \\
\boldsymbol{\Delta}_{u}{ }^{*}=\hat{y}_{\Delta_{u}}^{\dagger} \otimes d_{u}^{\dagger}, & & \boldsymbol{\Delta}_{\boldsymbol{d}}{ }^{*}=\hat{y}_{\Delta_{d}}^{\dagger} \otimes d_{d}^{\dagger}, \\
\boldsymbol{\Delta}_{\boldsymbol{L}}{ }^{*} & =\hat{y}_{\Delta_{L}}^{\dagger} \otimes d_{L}^{\dagger}, & \mathbf{K}=\hat{y}_{S}^{\dagger} \otimes s^{\dagger}, \\
\mathbf{\Omega}^{*}=\hat{y}_{\Omega} \otimes \omega, & \mathbf{\Upsilon}_{\boldsymbol{R}}^{\dagger}=\hat{y}_{M} \otimes \mathbf{M}_{R} .
\end{array}
$$

In these formulas the two component columns $h_{\nu, e, u, d}$ (in the weak isospin indices) are chosen in the same way as it was done in [5] (hereafter $v$ is an arbitrary complex constant of the dimension of the mass),

$$
\begin{array}{ll}
h_{\nu}=\left(\begin{array}{l}
v \\
0
\end{array}\right), & h_{e}=\left(\begin{array}{l}
0 \\
v
\end{array}\right), \\
h_{u}=\left(\begin{array}{l}
v \\
0
\end{array}\right), & h_{d}=\left(\begin{array}{l}
0 \\
v
\end{array}\right),
\end{array}
$$

and the three component columns $d_{u, d, L}$ (in the color indices) we choose as follows: 


$$
d_{u}=\left(\begin{array}{l}
v \\
0 \\
0
\end{array}\right), \quad d_{d}=\left(\begin{array}{l}
0 \\
v \\
0
\end{array}\right), \quad d_{L}=\left(\begin{array}{l}
0 \\
0 \\
v
\end{array}\right) .
$$

The quantity $s$ is the complex 3 by 2 matrix (in both color and the weak isospin indices),

$$
s=\left(\begin{array}{cc}
v & 0 \\
0 & 0 \\
0 & 0
\end{array}\right)
$$

$\omega$ is the complex number, which we set to $v$, and the dimensionful constant $\mathrm{M}_{R}$ sets the Majorana mass scale for the right-handed neutrinos, which is needed for the seesaw mechanism. The quantities $\hat{Y}_{u}, \hat{Y}_{d}, \hat{y}_{u}, \hat{y}_{d}, \hat{y}_{\Delta_{u}}, \hat{y}_{\Delta_{d}}, \hat{y}_{\Delta_{L}}, \hat{y}_{S}$, and $\hat{y}_{M}$ are arbitrary (dimensionless) complex 3 by 3 Yukawa matrices that act on the generation index. The tilde indicates charge conjugated weak isospin doublets, e.g., $\tilde{h}_{\nu}=\sigma_{2} h_{\nu}^{*}$, where $\sigma_{2}$ stands for the second Pauli matrix.

Considering the fluctuations (3.1) of the Dirac operator one can see that in order to construct the fluctuated Dirac operator, $\mathcal{D}$, one has replace the constant matrices in (3.2) by the matrix valued functions according to the following rule:

$$
\begin{array}{rlrlrl}
\tilde{h}_{\nu} & \rightarrow \tilde{H} & h_{e} \rightarrow H & \tilde{h}_{u} \rightarrow \tilde{H} & h_{d} \rightarrow H \\
d_{u} & \rightarrow \Delta_{u} & d_{d} \rightarrow \Delta_{d} & d_{L} \rightarrow \Delta_{L} & \\
s & \rightarrow S & \omega & \rightarrow \Omega . & &
\end{array}
$$

Note that upon the fluctuations of the Dirac operator $\mathrm{M}_{R}$ remains a constant; i.e., it does not transform into a field.

By definition the gauge subgroups $S U(2)$ and $S U(3)$ are represented on the weak isospin fermionic doublets and color fermionic triplets as a left multiplication by the unitary matrices $U_{S U(2)}$ and $U_{S U(3)}$, respectively, ${ }^{4}$

$[$ ferm doublet $] \rightarrow U_{S U(2)} \cdot[$ ferm doublet $] ;$

$$
[\text { ferm triplet }] \rightarrow U_{S U(3)} \cdot[\text { ferm triplet }],
$$

while the gauge fields transform upon the adjoint representation of the gauge group. The transformation law of the scalar fields that is presented below maintains the gauge invariance of the fermionic action upon the simultaneous gauge transformation of the fermionic multiplets, gauge and scalar fields. In what follows $Y$ stands for the Abelian hypercharge of a given multiplet, which describes the action of the $U(1)$ gauge subgroup.

\footnotetext{
${ }^{4} \mathrm{We}$ assume that the components of the weak isospin fermionic doublets and the color fermionic triplets are combined into columns. Note that antiquarks and antileptons are transformed by the complex conjugated matrices.
}

The scalar doublet $H$ is nothing but the Higgs field of the minimal standard model, which transforms as follows:

$H=\left[\begin{array}{c}H^{\text {up }} \\ H^{\text {down }}\end{array}\right] \underset{S U(2) \times S U(3)}{\longrightarrow} U_{S U(2)} \cdot H ; \quad \mathrm{Y}_{H}=1$.

The field $\tilde{H}$ transforms as $H$ under the $S U(2)$ transformations; however it has the opposite hypercharge,

$\tilde{H}=\left[\begin{array}{c}\left(H^{\text {down }}\right)^{*} \\ -\left(H^{\text {up }}\right)^{*}\end{array}\right] \underset{S U(2) \times S U(3)}{\longrightarrow} U_{S U(2)} \cdot \tilde{H} ; \quad \mathrm{Y}_{\tilde{H}}=-1$.

For each of the three fields $\Delta_{u}, \Delta_{d}$, and $\Delta_{L}$ the transformation law reads

$$
\begin{aligned}
\Delta_{u, d, L} & =\left[\begin{array}{c}
\Delta_{u, d, L}^{\mathrm{red}} \\
\Delta_{u, d, L}^{\mathrm{green}} \\
\Delta_{u, d, L}^{\text {blue }}
\end{array}\right] \underset{S U(2) \times S U(3)}{\stackrel{\longrightarrow}{\text { lue }}} U_{S U(3)} \cdot \Delta_{u, d, L} ; \\
\mathrm{Y}_{\Delta_{u}} & =\mathrm{Y}_{\Delta_{d}}=\mathrm{Y}_{\Delta_{L}}=\frac{4}{3} .
\end{aligned}
$$

The field $S$ carries both color and weak isospin indices and transforms in the following way:

$$
\begin{aligned}
S & =\left[\begin{array}{cc}
S^{\text {up red }} & S^{\text {down red }} \\
S^{\text {up green }} & S^{\text {down green }} \\
S^{\text {up blue }} & S^{\text {down blue }}
\end{array}\right] \underset{S U(2) \times S U(3)}{\longrightarrow} U_{S U(3)}^{-1 \mathrm{~T}} \cdot S \cdot U_{S U(2)}^{-1}, \\
\mathrm{Y}_{S} & =-\frac{1}{3}
\end{aligned}
$$

The last field $\Omega$ is the $S U(2) \times S U(3)$ singlet, and it transforms nontrivially just under the $U(1)$ transformations,

$$
\Omega \underset{S U(2) \times S U(3)}{\longrightarrow} \Omega, \quad \mathrm{Y}_{\Omega}=-2 .
$$

In the next section we compute the bosonic spectral action.

\section{BOSONIC SPECTRAL ACTION}

The aim of this section is to calculate the bosonic spectral action, ${ }^{5}$

$$
\begin{aligned}
S_{B} & \equiv \operatorname{Tr} \chi\left(\frac{\mathcal{D}^{2}}{\Lambda^{2}}\right) \\
& \simeq \Lambda^{4} f_{0} a_{0}+\Lambda^{2} f_{2} a_{2}+\Lambda^{0} f_{4} a_{4}+O\left(\frac{1}{\Lambda^{2}}\right),
\end{aligned}
$$

\footnotetext{
${ }^{5}$ In the present paper we exploit the standard definition of the bosonic spectral action, which is based on the introduction of the ultraviolet cutoff. Other definitions based, e.g., on the $\zeta$-function regularization are also possible [27].
} 
where $\chi$ is some cutoff function, $f_{0}, f_{2}, f_{4}$ are the first three momenta of its Fourier transform, and $a_{0}, a_{2}$, and $a_{4}$ are the first three nontrivial heat kernel coefficients on the manifold without boundary. The "fluctuated" (or covariant) Dirac operator is given by

$$
\mathcal{D}=\mathrm{i} \gamma^{\mu} \nabla_{\mu}+\gamma_{5} \otimes M
$$

where the covariant derivative $\nabla_{\mu}$ involves the gauge and the Levi-Civita spin connections, while the 96 by 96 matrix $M$ is nothing but the fluctuated version of $D_{F}$, which is obtained from (2.17) via the prescription (3.6).

Comment: We notice that the asymptotic expansion (4.1) correctly describes the behavior of the trace in the left-hand side of (4.1) at the energies below the cutoff scale $\Lambda$, while the high momenta behavior of the bosonic spectral action is drastically different [28]: high momenta bosons do not propagate; see also [29]. Physically it means that this model becomes strongly coupled at the energies above $\Lambda$ in both $U(1), S U(2)$, and $S U(3)$ sectors. A similar high energy phase transition has been considered beyond the scope of the noncommutative geometry; see, e.g., [30,31]. In what follows we do not discuss the high momenta regime and the mentioned above effects, so from now on the ansatz in the right-hand side of (4.1) is identified with the definition of the bosonic spectral action.

We emphasize that the gauge content of this formalism is identical to the one of [5]; therefore, if one sets $\Delta_{u, d, L}=0$, $\Omega=0$, and $S=0$ our operator $\mathcal{D}$ coincides with the one of [5]; hence it is sufficient to calculate the difference,

$$
S_{B}-\left.S_{B}\right|_{\Delta_{u, d, L}=0, \Omega=0, S=0} .
$$

\section{A. Computational simplifications}

The structure of the heat kernel coefficients on manifolds without boundaries is very well known (see, e.g., [32]), and one can easily see that the scalar fields can contribute to $a_{2}$ through the combination,

$$
a_{2}^{\text {contrib }}=\frac{1}{16 \pi^{2}} \int d^{4} x \sqrt{g} \operatorname{tr}(E),
$$

and to $a_{4}$ through the combination,

$$
a_{4}^{\text {contrib }}=\frac{1}{16 \pi^{2}} \frac{1}{360} \int d^{4} x \sqrt{g} \operatorname{tr}\left(-60 R E+180 E^{2}\right),
$$

where by definition

$$
E \equiv-D^{2}-\nabla^{2}
$$

and $R$ stands for a scalar curvature.

Note that the $a_{2}$ contribution cannot contain covariant derivatives of the scalar field: the simplest scalar contribution that involves the scalar fields and their covariant derivatives has the canonical dimension 3, while the integrand in (4.4) must have the canonical dimension 2. Therefore, to compute $a_{2}^{\text {contrib }}$ is sufficient to neglect the dependence of scalars on coordinates.

Now let us focus on the $a_{4}$ contribution. The computation of the scalar contribution to $a_{4}$ drastically simplifies, when the Dirac operator transforms in a homogeneous way upon the local Weyl transformation of the metric tensor and of the scalar fields. Even though the Dirac operator $\mathcal{D}$ does not exhibit this property (since it contains the constant Majorana mass terms for the right-handed neutrinos) one can write

$$
\operatorname{Tr} \chi\left(\frac{\mathcal{D}^{2}}{\Lambda^{2}}\right)=\left.\operatorname{Tr} \chi\left(\frac{\tilde{\mathcal{D}}^{2}}{\Lambda^{2}}\right)\right|_{\sigma=\mathrm{M}_{R}},
$$

where the "intermediate" Dirac operator $\tilde{\mathcal{D}}$ is obtained from $\mathcal{D}$ via the replacement of the constant $\mathrm{M}_{R}$ by the scalar field $\sigma$. This field has no gauge indices and it has already been considered in the context of the model to fix the Higgs mass in [12]. We emphasize that for the scope of the present article this field is needed at the intermediate step only, and by the end of the day it will be replaced by the constant $\mathrm{M}_{R}$.

Upon the local Weyl transformation

$$
\begin{aligned}
& g_{\mu \nu} \rightarrow e^{2 \phi} g_{\mu \nu}, \quad H \rightarrow e^{-\phi} H, \quad \Delta_{u, d, L} \rightarrow e^{-\phi} \Delta_{u, d, L}, \\
& S \rightarrow e^{-\phi} S, \quad \Omega \rightarrow e^{-\phi} \Omega, \quad \sigma \rightarrow e^{-\phi} \sigma,
\end{aligned}
$$

where $\phi$ is an arbitrary function of $x$, the intermediate Dirac operator $\tilde{\mathcal{D}}$ transforms in a homogeneous way,

$$
\tilde{\mathcal{D}} \rightarrow e^{-\frac{5 \phi}{2}} \tilde{\mathcal{D}} e^{\frac{3 \phi}{2}},
$$

and one can easily check (using the method of conformal variations, see for example [32]) that the fourth heat kernel coefficient that is associated with $\tilde{D}^{2}$ is Weyl invariant.

On the other side all heat kernel coefficients are gauge invariant. The only Weyl and gauge invariant combination of scalar fields of dimension 4 that involves the derivatives is ${ }^{6}$

$$
\begin{aligned}
\operatorname{tr} & {\left[D_{\mu}(\text { scalar field })^{\dagger} D^{\mu}(\text { scalar field })\right] } \\
& -\frac{1}{6} R \operatorname{tr}\left[(\text { scalar field })^{\dagger}(\text { scalar field })\right] ;
\end{aligned}
$$

thus it is sufficient to compute the coefficient in front of $R$ (scalar field $)^{\dagger}$ (scalar field), while the kinetic term, which contains all the covariant derivatives $D_{\mu}$, can be restored from (4.10). Note that for such a computation it is sufficient to consider constant scalar fields, $\partial_{\mu}($ scalar $)=0$, and set the gauge connection to 0 . Since the same simplification is

\footnotetext{
${ }^{6}$ The trace is needed since we are dealing with the matrix valued scalar fields like $S$.
} 
applicable for the $a_{2}$ contribution, let us assume it for a while. Using the well-known Lichnerowics formula one can easily check that in our "simplified" regime the endomorphism $E$, which enters in (4.4) and (4.5), equals to

$$
E=-M^{2} \otimes 1_{4}^{\mathrm{s}}+\frac{R}{4} \otimes 1_{384}
$$

hence

$$
E^{2}=M^{4} \otimes 1_{4}^{\mathrm{s}}+\frac{R^{2}}{16} \otimes 1_{384}-\left(\frac{R}{2}\right) \cdot M^{2} \otimes 1_{4}^{\mathrm{s}},
$$

so the calculation of the new terms of the bosonic spectral action is reduced to an algebraic exercise: one has to calculate $\operatorname{tr} M^{2}$ and $\operatorname{tr} M^{4}$. We recall that all the terms that disappeared because of our simplification can be recovered via the Weyl and the gauge invariance of $a_{4}$.

\section{B. Relevant traces}

One can check by a direct computation using, e.g., Maple, the following formulas:

$$
\begin{aligned}
\operatorname{tr} M^{2}= & 2 y_{1} \sigma^{2}+4 y_{2}\left(\Omega^{*} \Omega\right)+4 y_{3}\left(\Delta_{u}^{\dagger} \Delta_{u}\right)+4 y_{4}\left(\Delta_{d}^{\dagger} \Delta_{d}\right) \\
& +8 y_{5}\left(\Delta_{L}^{\dagger} \Delta_{L}\right)+12 y_{6}\left(H^{\dagger} H\right)+4 y_{7} \operatorname{tr}\left(S^{\dagger} S\right),
\end{aligned}
$$

and

$$
\begin{aligned}
\operatorname{tr} M^{4}= & 2 z_{1} \sigma^{4}+4 z_{2}\left(\Omega^{*} \Omega\right)^{2}+4 z_{3}\left(\Delta_{u}^{\dagger} \Delta_{u}\right)^{2}+4 z_{4}\left(\Delta_{d}^{\dagger} \Delta_{d}\right)^{2}+8 z_{5}\left(\Delta_{L}^{\dagger} \Delta_{L}\right)^{2}+12 z_{6}\left(H^{\dagger} H\right)^{2}+8 z_{7}\left(\Delta_{u}^{\dagger} \Delta_{u}\right)\left(\Omega^{*} \Omega\right) \\
& +8 z_{8}\left(\Delta_{d}^{\dagger} \Delta_{d}\right)\left(\Omega^{*} \Omega\right)+16 z_{9}\left(\Delta_{u}^{\dagger} \Delta_{u}\right)\left(H^{\dagger} H\right)+16 z_{10}\left(\Delta_{d}^{\dagger} \Delta_{d}\right)\left(H^{\dagger} H\right)+16 z_{11}\left(\Delta_{L}^{\dagger} \Delta_{L}\right)\left(H^{\dagger} H\right) \\
& +\left[8 z_{12}\left(\Delta_{d}^{\dagger} \Delta_{L}\right)\left(H^{\dagger} H\right)+8 z_{13}\left(\Delta_{u}^{\dagger} \Delta_{L}\right)\left(H^{\dagger} H\right)+\text { c.c. }\right]+8 z_{14}\left(\Omega^{*} \Omega\right)\left(H^{\dagger} H\right)+8 z_{15}\left(\Delta_{u}^{\dagger} \Delta_{u}\right) \sigma^{2} \\
& +8 z_{16}\left(\Omega^{*} \Omega\right) \sigma^{2}+8 z_{17}\left(H^{\dagger} H\right) \sigma^{2}+4 z_{18} \operatorname{tr}\left(S^{\dagger} S\right)^{2}+8 z_{19}\left(H^{\dagger} H\right) \operatorname{tr}\left(S^{\dagger} S\right)+8 z_{20}(S \tilde{H})^{\dagger}(S \tilde{H})+8 z_{21}(S H)^{\dagger}(S H) \\
& +8 z_{22}\left(\Delta_{u}^{\mathrm{T}} S\right)\left(\Delta_{u}^{\mathrm{T}} S\right)^{\dagger}+8 z_{23}\left(\Delta_{L}^{\dagger} \Delta_{L}\right) \operatorname{tr}\left(S^{\dagger} S\right)+\left[8 z_{24}\left(\tilde{H}^{\mathrm{T}} S^{\mathrm{T}} \Delta_{u}\right) \sigma+8 z_{25}\left(H^{\mathrm{T}} S^{\mathrm{T}} \Delta_{u} \Omega\right)+\text { c.c }\right],
\end{aligned}
$$

where the constants $y_{1}, \ldots, y_{7}, z_{1}, \ldots, z_{25}$ depend on the Yukawa couplings as follows:

$$
\begin{aligned}
& y_{1} \equiv \operatorname{tr}\left(\hat{y}_{M} \hat{y}_{M}^{\dagger}\right), \quad y_{2} \equiv \operatorname{tr}\left(\hat{y}_{\Omega} \hat{y}_{\Omega}^{\dagger}\right), \quad y_{3} \equiv \operatorname{tr}\left(\hat{y}_{\Delta_{u}} \hat{y}_{\Delta_{u}}^{\dagger}\right), \quad y_{4} \equiv \operatorname{tr}\left(\hat{y}_{\Delta_{d}} \hat{y}_{\Delta_{d}}^{\dagger}\right), \quad y_{5} \equiv \operatorname{tr}\left(\hat{y}_{\Delta_{L}} \hat{y}_{\Delta_{L}}^{\dagger}\right), \\
& y_{6} \equiv \operatorname{tr}\left(\left[\hat{y}_{u} \hat{y}_{u}^{\dagger}\right]+\left[\hat{y}_{d} \hat{y}_{d}^{\dagger}\right]+\frac{1}{3}\left[\hat{Y}_{u} \hat{Y}_{u}^{\dagger}\right]+\frac{1}{3}\left[\hat{Y}_{d} \hat{Y}_{d}^{\dagger}\right]\right), \quad y_{7} \equiv \operatorname{tr}\left(\hat{y}_{S} \hat{y}_{S}^{\dagger}\right), \\
& z_{1} \equiv \operatorname{tr}\left(\hat{y}_{M} \hat{y}_{M}^{\dagger}\right)^{2}, \quad z_{2} \equiv \operatorname{tr}\left(\hat{y}_{\Omega} \hat{y}_{\Omega}^{\dagger}\right)^{2}, \quad z_{3} \equiv \operatorname{tr}\left(\hat{y}_{\Delta_{u}} \hat{y}_{\Delta_{u}}^{\dagger}\right)^{2}, \quad z_{4} \equiv \operatorname{tr}\left(\hat{y}_{\Delta_{d}} \hat{y}_{\Delta_{d}}^{\dagger}\right)^{2}, \quad z_{5} \equiv \operatorname{tr}\left(\hat{y}_{\Delta_{L}} \hat{y}_{\Delta_{L}}^{\dagger}\right)^{2}, \\
& z_{6} \equiv \operatorname{tr}\left(\left[\hat{y}_{u} \hat{y}_{u}^{\dagger}\right]^{2}+\left[\hat{y}_{d} \hat{y}_{d}^{\dagger}\right]^{2}+\frac{1}{3}\left[\hat{Y}_{u} \hat{Y}_{u}^{\dagger}\right]^{2}+\frac{1}{3}\left[\hat{Y}_{d} \hat{Y}_{d}^{\dagger}\right]^{2}\right), \quad z_{7} \equiv \operatorname{tr}\left(\hat{y}_{\Omega} \hat{y}_{\Omega}^{\dagger}\right)\left(\hat{y}_{\Delta_{u}}^{\dagger} \hat{y}_{\Delta_{u}}\right), \quad z_{8} \equiv \operatorname{tr}\left(\hat{y}_{\Omega}^{\dagger} \hat{y}_{\Omega}\right)\left(\hat{y}_{\Delta_{d}}^{\mathrm{T}} \hat{y}_{\Delta_{d}}^{*}\right), \\
& z_{9} \equiv \frac{1}{2} \operatorname{tr}\left[\left(\hat{y}_{u} \hat{y}_{u}^{\dagger}\right)\left(\hat{y}_{\Delta_{u}} \hat{y}_{\Delta_{u}}^{\dagger}\right)+\left(\hat{Y}_{u} \hat{Y}_{u}^{\dagger}\right)\left(\hat{y}_{\Delta_{u}}^{\dagger} \hat{y}_{\Delta_{u}}\right)\right], \quad z_{10} \equiv \frac{1}{2} \operatorname{tr}\left[\left(\hat{y}_{d} \hat{y}_{d}^{\dagger}\right)\left(\hat{y}_{\Delta_{d}} \hat{y}_{\Delta_{d}}^{\dagger}\right)+\left(\hat{Y}_{d} \hat{Y}_{d}^{\dagger}\right)\left(\hat{y}_{\Delta_{d}}^{\dagger} \hat{y}_{\Delta_{d}}\right)\right], \\
& z_{11} \equiv \frac{1}{2} \operatorname{tr}\left[\left(\hat{y}_{u}^{\dagger} \hat{y}_{u}\right)\left(\hat{y}_{\Delta_{L}} \hat{y}_{\Delta_{L}}^{\dagger}\right)+\left(\hat{y}_{d}^{\dagger} \hat{y}_{d}\right)\left(\hat{y}_{\Delta_{L}} \hat{y}_{\Delta_{L}}^{\dagger}\right)+\left(\hat{Y}_{u}^{\dagger} \hat{Y}_{u}\right)\left(\hat{y}_{\Delta_{L}}^{\dagger} \hat{y}_{\Delta_{L}}\right)+\left(\hat{Y}_{d}^{\dagger} \hat{Y}_{d}\right)\left(\hat{y}_{\Delta_{L}}^{\dagger} \hat{y}_{\Delta_{L}}\right)\right], \quad z_{12} \equiv \operatorname{tr}\left(\hat{y}_{\Delta_{d}}^{\dagger} \hat{y}_{d} \hat{y}_{\Delta_{L}} \hat{Y}_{d}^{\dagger}\right), \\
& z_{13} \equiv \operatorname{tr}\left(\hat{y}_{\Delta_{u}}^{\dagger} \hat{y}_{u} \hat{y}_{\Delta_{L}} \hat{Y}_{u}^{\dagger}\right), \quad z_{14} \equiv \operatorname{tr}\left[\left(\hat{y}_{\Omega} \hat{y}_{\Omega}^{\dagger}\right)\left(\hat{Y}_{u} \hat{Y}_{u}^{\dagger}\right)+\left(\hat{y}_{\Omega}^{\dagger} \hat{y}_{\Omega}\right)\left(\hat{Y}_{d}^{*} \hat{Y}_{d}^{\mathrm{T}}\right)\right], \quad z_{15} \equiv \frac{1}{2} \operatorname{tr}\left[\left(\hat{y}_{M}^{\dagger} y_{M}\right)\left(\hat{y}_{\Delta_{u}}^{\mathrm{T}} \hat{y}_{\Delta_{u}}^{*}\right)+\left(\hat{y}_{M} y_{M}^{\dagger}\right)\left(\hat{y}_{\Delta_{u}}^{\dagger} \hat{y}_{\Delta_{u}}\right)\right], \\
& z_{16} \equiv \frac{1}{2} \operatorname{tr}\left[\left(\hat{y}_{\Omega} \hat{y}_{\Omega}^{\dagger}\right)\left(\hat{y}_{M} \hat{y}_{M}^{\dagger}\right)+\left(\hat{y}_{\Omega}^{*} \hat{y}_{\Omega}^{\mathrm{T}}\right)\left(\hat{y}_{M}^{\dagger} \hat{y}_{M}\right)\right], \quad z_{17} \equiv \operatorname{tr}\left(\hat{y}_{M} \hat{y}_{M}^{\dagger}\right)\left(\hat{Y}_{u} \hat{Y}_{u}^{\dagger}\right), \quad z_{18} \equiv \operatorname{tr}\left(\hat{y}_{S} \hat{y}_{S}^{\dagger}\right)^{2}, \quad z_{19} \equiv \operatorname{tr}\left[\left(\hat{y}_{S} \hat{y}_{S}^{\dagger}\right)\left(\hat{y}_{u}^{*} \hat{y}_{u}^{\mathrm{T}}\right)\right], \\
& z_{20} \equiv \operatorname{tr}\left[\left(\hat{y}_{S}^{\dagger} \hat{y}_{S}\right)\left(\hat{Y}_{u}^{\dagger} \hat{Y}_{u}\right)\right], \quad z_{21} \equiv \operatorname{tr}\left[\left(\hat{y}_{S}^{\dagger} \hat{y}_{S}\right)\left(\hat{Y}_{d}^{\dagger} \hat{Y}_{d}\right)\right], \quad z_{22} \equiv \operatorname{tr}\left[\left(\hat{y}_{S} \hat{y}_{S}^{\dagger}\right)\left(\hat{y}_{\Delta_{u}}^{*} \hat{y}_{\Delta_{u}}^{\mathrm{T}}\right)\right], \quad z_{23} \equiv \operatorname{tr}\left[\left(\hat{y}_{S}^{\dagger} \hat{y}_{S}\right)\left(\hat{y}_{\Delta_{L}}^{\dagger} \hat{y}_{\Delta_{L}}\right)\right], \\
& z_{24} \equiv \operatorname{tr}\left(\hat{y}_{\Delta_{u}} \hat{y}_{M} \hat{Y}_{u}^{*} \hat{y}_{S}^{\mathrm{T}}\right), \quad z_{25} \equiv \operatorname{tr}\left(\hat{y}_{\Delta_{y}} \hat{y}_{\Omega} \hat{Y}_{d}^{*} \hat{y}_{S}^{\mathrm{T}}\right) \text {. }
\end{aligned}
$$

\section{The full bosonic spectral action}

Substituting (4.13), (4.14), (4.11), and (4.12) in (4.4) and (4.5), recovering the dependence on the derivatives and on the gauge fields according to (4.10), and setting $\sigma=\mathrm{M}_{R}$ we arrive at the following answer for the new terms in the bosonic spectral action: 


$$
\begin{aligned}
S_{B}= & \left.S_{B}\right|_{\Delta_{u, d, S}=0, \Omega=0, S=0}+\int d^{4} x \sqrt{g^{\mathrm{E}}}\left\{-f_{2} \Lambda^{2}\left(\frac{y_{2}}{\pi^{2}} \Omega^{*} \Omega+\frac{y_{3}}{\pi^{2}} \Delta_{u}^{\dagger} \Delta_{u}+\frac{y_{4}}{\pi^{2}} \Delta_{d}^{\dagger} \Delta_{d}+\frac{2 y_{5}}{\pi^{2}} \Delta_{L}^{\dagger} \Delta_{L}+\frac{y_{7}}{\pi^{2}} \operatorname{tr} S^{\dagger} S\right)\right. \\
& +f_{4}\left\{\frac{y_{2}}{2 \pi^{2}}\left(D_{\mu} \Omega^{*} D^{\mu} \Omega-\frac{R}{6} \Omega^{*} \Omega\right) \frac{y_{3}}{2 \pi^{2}}\left(D_{\mu} \Delta_{u}^{\dagger} D^{\mu} \Delta_{u}-\frac{R}{6} \Delta_{u}^{\dagger} \Delta_{u}\right)+\frac{y_{4}}{2 \pi^{2}}\left(D_{\mu} \Delta_{d}^{\dagger} D^{\mu} \Delta_{d}-\frac{R}{6} \Delta_{d}^{\dagger} \Delta_{d}\right)\right. \\
& +\frac{y_{5}}{\pi^{2}}\left(D_{\mu} \Delta_{L}^{\dagger} D^{\mu} \Delta_{L}-\frac{R}{6} \Delta_{L}^{\dagger} \Delta_{L}\right)+\frac{y_{7}}{2 \pi^{2}} \operatorname{tr}\left(D_{\mu} S^{\dagger} D^{\mu} S-\frac{R}{6} S^{\dagger} S\right)+\frac{1}{2 \pi^{2}} z_{2}\left(\Omega^{*} \Omega^{2}+\frac{1}{2 \pi^{2}} z_{3}\left(\Delta_{u}^{\dagger} \Delta_{u}\right)^{2}+\frac{1}{2 \pi^{2}} z_{4}\left(\Delta_{d}^{\dagger} \Delta_{d}\right)^{2}\right. \\
& +\frac{1}{\pi^{2}} z_{5}\left(\Delta_{L}^{\dagger} \Delta_{L}\right)^{2}+\frac{1}{\pi^{2}} z_{7}\left(\Delta_{u}^{\dagger} \Delta_{u}\right)\left(\Omega^{*} \Omega\right)+\frac{1}{\pi^{2}} z_{8}\left(\Delta_{d}^{\dagger} \Delta_{d}\right)\left(\Omega^{*} \Omega\right)+\frac{2}{\pi^{2}} z_{9}\left(\Delta_{u}^{\dagger} \Delta_{u}\right)\left(H^{\dagger} H\right)+\frac{2}{\pi^{2}} z_{10}\left(\Delta_{d}^{\dagger} \Delta_{d}\right)\left(H^{\dagger} H\right) \\
& +\frac{2}{\pi^{2}} z_{11}\left(\Delta_{L}^{\dagger} \Delta_{L}\right)\left(H^{\dagger} H\right)+\frac{1}{\pi^{2}}\left[z_{12}\left(\Delta_{d}^{\dagger} \Delta_{L}\right)\left(H^{\dagger} H\right)+z_{13}\left(\Delta_{u}^{\dagger} \Delta_{L}\right)\left(H^{\dagger} H\right)+\mathrm{c} . \mathrm{c} .\right]+\frac{1}{\pi^{2}} z_{14}\left(\Omega^{*} \Omega\right)\left(H^{\dagger} H\right)+\frac{1}{\pi^{2}} z_{15}\left(\Delta_{u}^{\dagger} \Delta_{u}\right) \mathrm{M}_{R}^{2} \\
& +\frac{1}{\pi^{2}} z_{16}\left(\Omega^{*} \Omega\right) \mathrm{M}_{R}^{2}+\frac{1}{2 \pi^{2}} z_{18}\left[\operatorname{tr}\left(S^{\dagger} S\right)\right]^{2}+\frac{1}{\pi^{2}} z_{19}\left(H^{\dagger} H\right) \operatorname{tr}\left(S^{\dagger} S\right)+\frac{1}{\pi^{2}} z_{20}(S \tilde{H})^{\dagger}(S \tilde{H})+\frac{1}{\pi^{2}} z_{21}(S H)^{\dagger}(S H) \\
& \left.+\frac{1}{\pi^{2}} z_{22}\left(\Delta_{u}^{\mathrm{T}} S\right)\left(\Delta_{u}^{\mathrm{T}} S\right)^{\dagger}+\frac{1}{\pi^{2}} z_{23}\left(\Delta_{L}^{\dagger} \Delta_{L}\right) \operatorname{tr}\left(S^{\dagger} S\right)+\frac{1}{\pi^{2}}\left[z_{24}\left(\tilde{H}^{\mathrm{T}} S^{\mathrm{T}} \Delta_{u}\right) \mathrm{M}_{R}+z_{25}\left(H^{\mathrm{T}} S^{\mathrm{T}} \Delta_{u} \Omega\right)+\mathrm{c} . \mathrm{c}\right]\right\} .
\end{aligned}
$$

This is the result of the spectral action computation with the new fields coming form the Clifford requirement grading.

\section{TOWARDS THE PHYSICAL ACTION}

In this section we discuss how to make our spectral action applicable in a physical context. In order to do this one has to carry out two important steps.

(i) Get rid of the redundant fermionic degrees of freedom.

(ii) Make the action Lorentzian.

The redundancy is usually solved projecting out the extra degrees of freedom [5,24,33], while the Euclidean vs Lorentzian issue has several ramifications (see, for example, [34-37]), but the usual method is to perform a Wick rotation. We have shown in [25] that the two issues are intimately related, and give a prescription on how to deal with them.

\section{A. General prescription: a review and discussion}

Now we briefly recall how the Wick rotation works following [25]. In order to pass from the Euclidean to a Lorentzian theory, each expression $F$ that involves the vierbeins $e_{\mu}^{a}$ has to be transformed according to the following rule:

$$
\text { Wick: } F\left[e_{\mu}^{0}, e_{\mu}^{j}\right] \rightarrow F\left[i e_{\mu}^{0}, e_{\mu}^{j}\right], \quad j=1,2,3 .
$$

As it was demonstrated in [25], upon the transformation (5.1) the Euclidean bosonic action $S_{\text {bos }}^{\mathrm{E}}$, which comes out from the first three nonzero heat kernel coefficients, perfectly transforms into the "textbook" Lorentzian action $S_{\text {bos }}^{\mathrm{M}}$, in particular,

$$
\text { Wick: } \begin{aligned}
\exp & \left(-S_{\text {bos }}^{\mathrm{E}}\left[\text { fields, } g_{\mu \nu}^{\mathrm{E}}\right]\right) \\
& \rightarrow \exp \left(\mathrm{i} S_{\text {bos }}^{\mathrm{M}}\left[\text { fields, } g_{\mu \nu}^{\mathrm{M}}\right]\right),
\end{aligned}
$$

where the metric tensors $g_{\mu \nu}^{\mathrm{E}}$ and $g_{\mu \nu}^{\mathrm{M}}$ have the signatures $\{+,+,+,+\}$ and $\{+,-,-,-\}$, respectively. We refer the reader to the quoted reference for the details.

A treatment of the fermionic action is more subtle, since the product space $\mathcal{H}$ contains extra degrees of freedom. Now we briefly recall what the problem is. The Hilbert space $\mathcal{H}$ of the almost commutative geometry has the following structure,

$$
\mathcal{H}=\operatorname{sp}(M) \otimes \mathcal{H}_{F}=\mathcal{H}_{L} \oplus \mathcal{H}_{R} \oplus \mathcal{H}_{L}^{c} \oplus \mathcal{H}_{R}^{c},
$$

where the subspaces $\mathcal{H}_{L}, \mathcal{H}_{R}$ by definition consist of the multiplets of the nonchiral four-component spinors that transform under the gauge transformations as the multiplets of the left-handed and right-handed chiral fermions of the standard model, while the subspaces $\mathcal{H}_{L}^{c}$ and $\mathcal{H}_{R}^{c}$ consist of the nonchiral four-component spinors fermions that transform under the gauge transformations as the charge conjugated multiplets of the chiral left and right fermions of the standard model. This doubling of the degrees of freedom is called in [25] the "mirror doubling." On the one side the action of the standard model does not contain any independent variables with the index "c," which indicates the charge conjugated field: the charge conjugated spinor is obtained from the original one via the charge conjugation operation [i.e., they are not independent variables; see (5.12) below]. This other doubling is called in [25] the charge conjugation doubling.

In order to get rid of the mirror doubling one has to extract the particles with the correct chirality. The left $\psi_{\mathcal{L}}$ and the right $\psi_{\mathcal{R}}$ chiral spinors are by definition the eigenstates of the left and the right chiral projectors,

$$
\psi_{\mathcal{L}}=\frac{1}{2}\left(1-\gamma^{5}\right) \psi_{\mathcal{L}}, \quad \psi_{\mathcal{R}}=\frac{1}{2}\left(1+\gamma^{5}\right) \psi_{\mathcal{R}} .
$$


In order to get rid of the redundant fermionic degrees of freedom with the wrong chirality one has to extract just left chiral fermions from $\mathcal{H}_{L}$ and $\mathcal{H}_{R}^{c}$ and just right chiral fermions from $\mathcal{H}_{R}$ and $\mathcal{H}_{L}^{c}$, where we took into account the fact that for the physical fermions, which live in Lorentzian space-time, the antiparticles have the opposite chirality with respect to the original particles. So the subspace $\mathcal{H}_{+}$of $\mathcal{H}$ that contains just the fermions with correct chiralities has the following structure:

$$
\mathcal{H}_{+}=\left(H_{L}\right)_{\mathcal{L}} \oplus\left(\mathcal{H}_{R}\right)_{\mathcal{R}} \oplus\left(\mathcal{H}_{L}^{c}\right)_{\mathcal{R}} \oplus\left(\mathcal{H}_{R}^{c}\right)_{\mathcal{L}}
$$

In the original paper [5] such an extraction was presented in the form

$$
P_{+} \mathcal{H}_{+}=\mathcal{H}_{+},
$$

where the projector $P_{+}$is defined via the grading as follows:

$$
P_{+}=\frac{1}{2}\left(1+\gamma^{5} \otimes \gamma_{F}^{\mathrm{st}}\right)
$$

In this formula $\gamma_{F}^{\text {st }}$ stands for the standard grading introduced in [5]. Since we are working with the different grading $\gamma_{F}$, in order to arrive at the correct subspace (5.5) the connection between the projector $P_{+}$and the grading $\gamma_{F}$ takes a slightly different form,

$$
P_{+}=\frac{1}{2}\left(1+\gamma^{5} \otimes(\mathrm{Q}-\mathrm{L}) \gamma_{F}\right)
$$

The Euclidean fermionic action introduced in [5], which is free of the mirror doubling, reads

$S_{F}^{\mathrm{E}}=\frac{1}{2} \int d^{4} x \sqrt{g^{\mathrm{E}}}\left(\mathcal{J} \Psi_{+}\right)^{\dagger} \mathcal{D} \Psi_{+}, \quad \Psi_{+} \in \mathcal{H}_{+}$.

As [25] shows, after the Wick rotation of the vierbeins (5.1) one obtains

$$
\text { Wick: } \begin{aligned}
\exp & \left(-S_{F}^{\mathrm{E}}\left[\text { spinors, } e_{\mu}^{a}\right]\right) \\
& \rightarrow \exp \left(\mathrm{i} S_{F}^{\mathrm{M}} \text { doubled }\left[\text { spinors, } e_{\mu}^{a}\right]\right),
\end{aligned}
$$

where the intermediate fermionic action $S_{F}^{\mathrm{M}}$ doubled is already Lorentz invariant. However, due to the charge conjugation doubling, it depends on twice more fermionic fields than is needed; it is not real and therefore it is not suitable for the canonical quantization. In order to complete a construction of the physical fermionic action one has to eliminate the charge conjugation doubling via the following identification of the variables in the action $S_{F}^{\mathrm{M}}$ doubled from the subspaces $\mathcal{H}_{L}^{c}$ and $\mathcal{H}_{R}^{c}$ with the variables from $\mathcal{H}_{L}$ and $\mathcal{H}_{R}$,

$$
\text { step 1: }\left\{\begin{array}{l}
\left(\psi_{L}^{c}\right)_{\mathcal{R}} \in \underbrace{\left(H_{L}^{c}\right)_{\mathcal{R}}}_{\subset H_{+}} \text {has to be identified with } C_{\mathrm{M}}\left(\psi_{L}\right)_{\mathcal{L}}, \quad\left(\psi_{L}\right)_{\mathcal{L}} \in \underbrace{\left(H_{L}\right)_{\mathcal{L}}}_{\subset H_{+}} \\
\left(\psi_{R}^{c}\right)_{\mathcal{L}} \in \underbrace{\left(H_{R}^{c}\right)_{\mathcal{L}}}_{\subset H_{+}} \text {has to be identified with } C_{\mathrm{M}}\left(\psi_{R}\right)_{\mathcal{R}}, \quad\left(\psi_{R}\right)_{\mathcal{R}} \in \underbrace{\left(H_{R}\right)_{\mathcal{R}}}_{\subset H_{+}},
\end{array}\right.
$$

where the operation $C_{\mathrm{M}}$ is the charge conjugation operation, which acts on the arbitrary spinor $\psi$ as follows:

$$
C_{\mathrm{M}} \psi=-\mathrm{i} \gamma^{2} \psi^{*}
$$

Note that in contrast to the Euclidean charge conjugation $J$ the operation $C_{\mathrm{M}}$ changes a chirality. We emphasize that the identification (5.11) makes sense after the Wick rotation to Lorentzian signature, since the quantities to be identified transform in the same way under the Lorentzian $S O(1,3)$ transformations rather than Euclidean $S O(4)$ rotations. After the global axial transformation of all the remaining spinors,

$$
\text { step 2: } \psi \rightarrow e^{-\frac{\mathrm{i} \pi}{4} \gamma^{5}} \psi
$$

one arrives ar the textbook form $S_{F}^{\mathrm{M}}$ of the fermionic action,

$$
\begin{aligned}
\text { step } 1+\operatorname{step} 2: & \exp \left(\mathrm{i} S_{F}^{\mathrm{M}} \text { doubled }\left[\text { spinors, } e_{\mu}^{a}\right]\right) \\
& \rightarrow \exp \left(\mathrm{i} S_{F}^{\mathrm{M}}\left[\text { phys spinors, } e_{\mu}^{a}\right]\right) .
\end{aligned}
$$

Following [25] we recall that the last step must be performed before the quantization: otherwise one gets an additional Pontryagin gauge action that comes out from the Abelian axial anomaly. Below we apply these prescriptions to our model and we find out a nontrivial outcome.

\section{B. This model}

Let us parametrize the elements of the Hilbert space $\mathcal{H}$ as follows:

$$
\Psi=\left(\mathbf{v}_{R}, \mathbf{e}_{R}, \mathbf{L}_{L}, \mathbf{u}_{R}, \mathbf{d}_{R}, \mathbf{Q}_{L}, \mathbf{v}_{R}^{c}, \mathbf{e}_{R}^{c}, \mathbf{L}_{L}^{c}, \mathbf{u}_{R}^{c}, \mathbf{d}_{R}^{c}, \mathbf{Q}_{L}^{c}\right)^{\mathrm{T}} .
$$

This is basically the parametrization (2.10) of the elements of $\mathcal{H}_{F}$; the change of typeface indicates that the elements of 
$\mathcal{H}$ are spinors, no longer complex numbers. In these notations $\mathbf{u}_{R}$ is a collection of four-component spinors that transforms upon the action of the gauge group as the right-handed quarks, $\mathbf{u}_{R}^{c}$ is an independent collection of four-component spinors that transforms upon the action of the gauge group as the charge conjugated right-handed quark field, and so on. The typical element of $\mathcal{H}_{+}$, which is constructed according to (5.5), then becomes

$$
\begin{aligned}
\Psi_{+}= & \left(\left[\mathbf{u}_{R}\right]_{\mathcal{R}},\left[\mathbf{d}_{R}\right]_{\mathcal{R}},\left[\mathbf{Q}_{L}\right]_{\mathcal{L}},\left[\mathbf{v}_{R}\right]_{\mathcal{R}},\left[\mathbf{e}_{R}\right]_{\mathcal{R}},\left[\mathbf{L}_{L}\right]_{\mathcal{L}},\right. \\
& {\left.\left[\mathbf{u}_{R}^{c}\right]_{\mathcal{L}},\left[\mathbf{d}_{R}^{c}\right]_{\mathcal{L}},\left[\mathbf{Q}_{L}^{c}\right]_{\mathcal{R}},\left[\mathbf{v}_{R}^{c}\right]_{\mathcal{L}},\left[\mathbf{e}_{R}^{c}\right]_{\mathcal{L}},\left[\mathbf{L}_{L}^{c}\right]_{\mathcal{R}}\right)^{\mathrm{T}} . }
\end{aligned}
$$

Composing the fermionic action (5.9), applying the Wick rotation procedure (5.1), removing the charge conjugation doubling according to the general prescription (5.11), and finally carrying out the axial transformation (5.13) we see that

$$
\text { Wick rotation }+ \text { dequadrupling: }-S_{F}^{\mathrm{E}} \rightarrow \mathrm{i} S_{F}^{\mathrm{M}} \text {, }
$$

where $S_{F}^{\mathrm{M}}$ is given by

$$
\begin{aligned}
S_{F}^{\mathrm{M}}= & \int d^{4} x \sqrt{-g^{\mathrm{M}}}\left\{\overline{i\left(\overline{\left.\mathbf{u}_{\mathcal{R}}\right)}\right.} \not \mathbf{u}_{\mathcal{R}}+\overline{i\left(\mathbf{d}_{\mathcal{R}}\right)} \bar{\nabla} \mathbf{d}_{\mathcal{R}}+i \overline{\left(\mathbf{Q}_{\mathcal{L}}\right)} \not \mathbf{\mathbf { Q } _ { \mathcal { L } }}+\overline{i\left(\mathbf{v}_{\mathcal{R}}\right)} \not \mathbf{v}_{\mathcal{R}}+\overline{i\left(\mathbf{e}_{\mathcal{R}}\right)} \not \mathbf{e}_{\mathcal{R}}+\overline{i\left(\mathbf{L}_{\mathcal{L}}\right)} \nabla \mathbf{L}_{\mathcal{L}}\right. \\
& -\left[\overline{\left(\mathbf{Q}_{\mathcal{L}}\right)}\left[\hat{y}_{u}^{\dagger} \otimes \tilde{H}\right] \mathbf{u}_{\mathcal{R}}+\overline{\left(\mathbf{Q}_{\mathcal{L}}\right)}\left[\hat{y}_{d}^{\dagger} \otimes H\right] \mathbf{d}_{\mathcal{R}}+\overline{\left(\mathbf{L}_{\mathcal{L}}\right)}\left[\hat{Y}_{u}^{\dagger} \otimes \tilde{H}\right] \mathbf{v}_{\mathcal{R}}+\overline{\left(\mathbf{L}_{\mathcal{L}}\right)}\left[\hat{Y}_{d}^{\dagger} \otimes H\right] \mathbf{e}_{\mathcal{R}}\right. \\
& \left.\left.+\frac{1}{2} \overline{\left(C_{\mathrm{M}} \mathbf{v}_{\mathcal{R}}\right)}\left[\hat{y}_{M}^{\dagger}\right] \mathbf{v}_{\mathcal{R}}+\overline{\left(C_{\mathrm{M}} \mathbf{e}_{\mathcal{R}}\right)}\left[\hat{y}_{\Omega}^{\dagger} \otimes \Omega^{*}\right] \mathbf{v}_{\mathcal{R}}+\text { c.c. }\right]\right\} .
\end{aligned}
$$

In this formula for an arbitrary spinor $\psi$ the bar stands for the Dirac conjugation, $\bar{\psi} \equiv \psi^{\dagger} \gamma^{0}$. Note that after the Wick rotation accompanied by the elimination of the fermionic quadrupling just the multiplets of the sructures $\left[\psi_{R}\right]_{\mathcal{R}}$ and $\left[\psi_{L}\right]_{\mathcal{L}}$ remain in the result; therefore we simplified the notations replacing them by $\psi_{\mathcal{R}}$ and $\psi_{\mathcal{L}}$, respectively.

We now come to an important point of this noncommutative geometric construction. Note that the fields $\Delta_{u}, \Delta_{d}$, $\Delta_{L}$, and $S$, which are present in the Dirac operator and hence in the bosonic spectral action, are absent in the fermionic action (5.18). Let us clarify what has happened. If one looks carefully at the structure $(\mathcal{J} \Psi)^{\dagger} \mathcal{D} \Psi, \Psi \in \mathcal{H}$ one immediately finds out that the mentioned fields always appear in interaction terms in the action (vertices) that involve spinors with unphysical chiralities. Therefore, when one restricts the fermions just to the "good-chirality" subspace $\mathcal{H}_{+}$, all these terms vanish. Indeed, the $S$ field enters in $(\mathcal{J} \Psi)^{\dagger} \mathcal{D} \Psi$, in particular, via the combination

$$
\left(J \mathbf{u}_{R}\right)^{\dagger}\left(\gamma^{5} \otimes \hat{y}_{S} \otimes S\right) \mathbf{L}_{L} .
$$

When one restricts $\Psi \in H_{+}$this expression turns into

$$
\begin{aligned}
& \left(J\left[\mathbf{u}_{R}\right]_{\mathcal{R}}\right)^{\dagger}\left(\gamma^{5} \otimes \hat{y}_{S} \otimes S\right)\left[\mathbf{L}_{L}\right]_{\mathcal{L}} \\
& \quad \equiv\left(J P_{\mathcal{R}} \mathbf{u}_{R}\right)^{\dagger}\left(\gamma^{5} \otimes \hat{y}_{S} \otimes S\right) P_{\mathcal{L}} \mathbf{L}_{L} \\
& \quad=\left(J \mathbf{u}_{R}\right)^{\dagger}\left(\gamma^{5} \otimes \hat{y}_{S} \otimes S\right)\left(P_{\mathcal{R}} P_{\mathcal{L}}\right) \mathbf{L}_{L}=0,
\end{aligned}
$$

where we took into account the fact that the chiral projectors $P_{\mathcal{L}}=\frac{1}{2}\left(1_{4}-\gamma^{5}\right)$ and $P_{\mathcal{R}}=\frac{1}{2}\left(1_{4}+\gamma^{5}\right)$ commute with the Euclidean charge conjugation $J$ and remain unchanged upon the Hermitian conjugation of matrices.
One can easily check that all other combinations that involve $S$ vanish according to the same mechanism.

Now let us see what has happened to the $\Delta_{u, d, L}$ fields. The $\Delta_{u}$ field enters in $(\mathcal{J} \Psi)^{\dagger} \mathcal{D} \Psi$ in particular through the expression

$$
\left(J \mathbf{u}_{R}^{c}\right)^{\dagger}\left(\gamma^{5} \otimes \hat{y}_{\Delta_{u}} \otimes \Delta_{u}\right) \mathbf{v}_{R} .
$$

Upon the restriction $\Psi \in \mathcal{H}_{+}$this expression turns into

$$
\begin{aligned}
& \left(J\left[\mathbf{u}_{R}^{c}\right]_{\mathcal{L}}\right)^{\dagger}\left(\gamma^{5} \otimes \hat{y}_{\Delta_{u}} \otimes \Delta_{u}\right)\left[\mathbf{v}_{R}\right]_{\mathcal{R}} \\
& \quad=\left(J P_{\mathcal{L}} \mathbf{u}_{R}^{c}\right)^{\dagger}\left(\gamma^{5} \otimes \hat{y}_{\Delta_{u}} \otimes \Delta_{u}\right) P_{\mathcal{R}} \mathbf{v}_{R} \\
& \quad=\left(J \mathbf{u}_{R}^{c}\right)^{\dagger}\left(\gamma^{5} \otimes \hat{y}_{\Delta_{u}} \otimes \Delta_{u}\right) P_{\mathcal{L}} P_{\mathcal{R}} \mathbf{v}_{R}=0 .
\end{aligned}
$$

One can easily check that all other terms, which involve the $\Delta$-fields, vanish as well in a similar way.

\section{CONCLUSIONS AND OUTLOOK}

Based on the purely algebraic idea to incorporate the Clifford structure in the finite dimensional spectral triple, proposed in [23], we arrive at a set of new scalar fields in the minimal version of the noncommutative standard model. Some of the new scalar fields (viz. $\Delta_{u, d, L}$ and $S$ ) carry both color and the weak isospin indices. The fields of such a kind are of interest in recent phenomenological research; in particular, the scalar leptoquarks are the case in Ref. [38].

We computed the new terms in the bosonic spectral action, which come out from these fields. Equation (4.16) is one of the main results of this article. The scalar-scalar couplings between the new fields and the Higgs field may 
improve the minimal noncommutative standard model from the phenomenological point of view: they give positive contributions to the beta function of the Higgs self-interaction quartic constant at the level of the one loop [39], what is needed to avoid the vacuum instability problem [40,41].

We did not discuss in detail in this paper the possible phenomenological consequences of these new terms. The whole approach to the standard model based on noncommutative geometry is now reaching the level to be confronted with phenomenology, and of course the scalar sector seems to be of paramount importance. The new fields discussed here may possibly be part of this, but more work is necessary in this direction.

The approach is interesting from the mathematical point of view as well. It turns out that some of the new fields (viz. $\Delta_{u, d, L}$ and $S$ ) are coupled to the spurious fermionic degrees of freedom, whose presence is due to the "product-based" construction of the almost commutative spectral triple. Therefore, this model exhibits a very peculiar property, which is another important result of this article. On the one side these fields do not enter in the physical (Minkowskian) fermionic Lagrangian (5.18), even though they appear in the Euclidean NCG Dirac operator. On the other side the physical (Wick rotated to the Lorentzian signature) bosonic spectral action keeps memory about these extra degrees of freedom: it depends on $\Delta_{u, d, L}$ and $S$. Therefore, the fermionic quadrupling in the spectral approach is not just the presence of the extra fermions to be projected out: by the end of the day it affects nontrivially the bosonic action of the model, without altering the fermionic action.

In this article we considered an evolution of the spectral approach from an algebraic point of view. There are other interesting mathematical directions that can be taken. In particular, we consider the manifold $\mathcal{M}$ without boundary; manifolds with boundaries have been considered within the spectral action formalism as well [42-44]. Recently another purely spectral feature has been discovered: parity anomaly on four-dimensional manifolds with boundaries $[45,46]$. It would be interesting to understand the role played by the parity anomaly in the context of the spectral action approach, and the issue deserves further scrutiny.

\section{ACKNOWLEDGMENTS}

We thank Francesco D'Andrea for several illuminating discussions that helped shape this paper, and clarifications of various conceptual and technical aspects of the Cliffordbased approach. F. L. acknowledges the support of the COST action QSPACE, the INFN Iniziativa Specifica GeoSymQFT, and the Spanish MINECO Grant No. MDM-2014-0369 of Institut de Ciències del Cosmos Universitat de Barcelona (ICCUB) (Unidad de Excelencia "Maria de Maeztu"). M. K. acknowledges the support of the Sao-Paulo Research Foundation (FAPESP), Grant No. 2015/05120-0.
[1] A. Connes, Noncommutative Geometry (Wiley, New York, 1994).

[2] G. Landi, An Introduction to Noncommutative Spaces and their Geometries (Springer, New York, 1997).

[3] P. Aschieri, M. Dimitrijevic, P. Kulish, F. Lizzi, and J. Wess, Noncommutative space-times: Symmetries in noncommutative geometry and field theory, Lect. Notes Phys. 774, 1 (2009).

[4] A. Connes and M. Marcolli, Noncommutative Geometry, Quantum Fields, and Motives (AMS, Providence, RI, 2007).

[5] A. H. Chamseddine, A. Connes, and M. Marcolli, Gravity and the standard model with neutrino mixing, Adv. Theor. Math. Phys. 11, 991 (2007).

[6] W. Van Suijlekom, Noncommutative Geometry and Particle Physics (Springer, New York, 2015).

[7] A. H. Chamseddine and A. Connes, The spectral action principle, Commun. Math. Phys. 186, 731 (1997).

[8] A. A. Andrianov and F. Lizzi, Bosonic spectral action induced from anomaly cancellation, J. High Energy Phys. 05 (2010) 057.

[9] A. A. Andrianov, M. A. Kurkov, and F. Lizzi, Spectral action, Weyl anomaly, and the Higgs-Dilaton potential, J. High Energy Phys. 10 (2011) 001.
[10] M. A. Kurkov and F. Lizzi, Higgs-dilaton Lagrangian from spectral regularization, Mod. Phys. Lett. A 27, 1250203 (2012).

[11] M. A. Kurkov and M. Sakellariadou, Spectral regularization: Induced gravity and the onset of inflation, J. Cosmol. Astropart. Phys. 01 (2014) 035.

[12] A. H. Chamseddine and A. Connes, Resilience of the spectral standard model, J. High Energy Phys. 09 (2012) 104.

[13] A. Devastato, F. Lizzi, and P. Martinetti, Grand symmetry, spectral action, and the Higgs mass, J. High Energy Phys. 01 (2014) 042.

[14] A. Devastato, F. Lizzi, and P. Martinetti, Higgs mass in noncommutative geometry, Fortschr. Phys. 62, 863 (2014).

[15] A. H. Chamseddine, A. Connes, and W. D. van Suijlekom, Inner fluctuations in noncommutative geometry without the first order condition, J. Geom. Phys. 73, 222 (2013).

[16] A. H. Chamseddine, A. Connes, and W. D. van Suijlekom, Beyond the spectral standard model: Emergence of PatiSalam unification, J. High Energy Phys. 11 (2013) 132.

[17] A. Devastato and P. Martinetti, Twisted spectral triple for the standard model and spontaneous breaking of the grand symmetry, Math. Phys. Anal. Geom. 20, 2 (2017). 
[18] A. H. Chamseddine, A. Connes, and W. D. van Suijlekom, Grand unification in the spectral Pati-Salam model, J. High Energy Phys. 11 (2015) 011.

[19] U. Aydemir, D. Minic, and T. Takeuchi, The Higgs mass and the emergence of new physics, Phys. Lett. B 724, 301 (2013).

[20] U. Aydemir, D. Minic, C. Sun, and T. Takeuchi, Higgs mass, superconnections, and the $\mathrm{TeV}$-scale left-right symmetric model, Phys. Rev. D 91, 045020 (2015).

[21] U. Aydemir, D. Minic, C. Sun, and T. Takeuchi, Pati-Salam unification from noncommutative geometry and the TeVscale $W_{R}$ boson, Int. J. Mod. Phys. A 31, 1550223 (2016).

[22] U. Aydemir, D. Minic, C. Sun, and T. Takeuchi, The $750 \mathrm{GeV}$ diphoton excess in unified $S U(2)_{L} \times S U(2)_{R} \times S U(4)$ models from noncommutative geometry, Mod. Phys. Lett. A 31, 1650101 (2016).

[23] F. D'Andrea and L. Dabrowski, The standard model in noncommutative geometry and Morita equivalence, J. Noncommut. Geom. 10, 551 (2016).

[24] F. Lizzi, G. Mangano, G. Miele, and G. Sparano, Fermion Hilbert space and fermion doubling in the noncommutative geometry approach to gauge theories, Phys. Rev. D 55, 6357 (1997).

[25] F. D'Andrea, M. A. Kurkov, and F. Lizzi, Wick rotation and fermion doubling in noncommutative geometry, Phys. Rev. D 94, 025030 (2016).

[26] A. Connes, On the spectral characterization of manifolds, J. Noncommut. Geom. 7, 1 (2013).

[27] M. A. Kurkov, F. Lizzi, M. Sakellariadou, and A. Watcharangkool, Spectral action with zeta function regularization, Phys. Rev. D 91, 065013 (2015).

[28] M. A. Kurkov, F. Lizzi, and D. Vassilevich, High energy bosons do not propagate, Phys. Lett. B 731, 311 (2014).

[29] N. Alkofer, F. Saueressig, and O. Zanusso, Spectral dimensions from the spectral action, Phys. Rev. D 91, 025025 (2015).

[30] V. A. Rubakov and S. V. Troitsky, Trends in grand unification: Unification at strong coupling and composite models, arXiv:0001213.

[31] A. A. Andrianov, D. Espriu, M. A. Kurkov, and F. Lizzi, Universal Landau Pole, Phys. Rev. Lett. 111, 011601 (2013).
[32] D. V. Vassilevich, Heat kernel expansion: User's manual, Phys. Rep. 388, 279 (2003).

[33] J. M. Gracia-Bondia, B. Iochum, and T. Schucker, The standard model in noncommutative geometry and fermion doubling, Phys. Lett. B 416, 123 (1998).

[34] K. v. d. Dungen, Krein spectral triples and the fermionic action, Math. Phys. Anal. Geom. 19, 4 (2016).

[35] N. Franco and M. Eckstein, An algebraic formulation of causality for noncommutative geometry, Classical Quantum Gravity 30, 135007 (2013).

[36] A. Devastato, S. Farnsworth, F. Lizzi, and P. Martinetti, Lorentz signature and twisted spectral triples, J. High Energy Phys. 03 (2018) 089.

[37] N. Bizi, C. Brouder, and F. Besnard, Space and time dimensions of algebras with applications to Lorentzian noncommutative geometry and quantum electrodynamics, arXiv:1611.07062.

[38] C. Patrignani et al. (Particle Data Group), Review of particle physics, Chin. Phys. C 40, 100001 (2016).

[39] M. E. Machacek and M. T. Vaughn, Two loop renormalization group equations in a general quantum field theory. 3 . Scalar quartic couplings, Nucl. Phys. B249, 70 (1985).

[40] J. Elias-Miro, J. R. Espinosa, G. F. Giudice, G. Isidori, A. Riotto, and A. Strumia, Higgs mass implications on the stability of the electroweak vacuum, Phys. Lett. B 709, 222 (2012).

[41] F. Bezrukov, M. Y. Kalmykov, B. A. Kniehl, and M. Shaposhnikov, Higgs boson mass and new physics, J. High Energy Phys. 10 (2012) 140.

[42] A. H. Chamseddine and A. Connes, Noncommutative geometric spaces with boundary: Spectral action, J. Geom. Phys. 61, 317 (2011).

[43] A. H. Chamseddine and A. Connes, Quantum Gravity Boundary Terms from Spectral Action, Phys. Rev. Lett. 99, 071302 (2007).

[44] B. Iochum, C. Levy, and D. Vassilevich, Spectral action for torsion with and without boundaries, Commun. Math. Phys. 310, 367 (2012).

[45] M. Kurkov and D. Vassilevich, Parity anomaly in four dimensions, Phys. Rev. D 96, 025011 (2017).

[46] M. Kurkov and D. Vassilevich, Gravitational parity anomaly with and without boundaries, J. High Energy Phys. 03 (2018) 072. 